\title{
On Generating New Amicable Pairs from Given Amicable Pairs
}

\author{
By Herman J. J. te Riele
}

\begin{abstract}
Methods are given for constructing new amicable pairs from given amicable pairs. By applying these methods to 1575 "mother" pairs known to the author, 1782 new amicable pairs were generated, so that the average "offspring" of these mother pairs is greater than 1 . By a second, much more restricted application of the methods to the 400 ) smallest "daughter" pairs, 88 new "granddaughter" pairs were generated.
\end{abstract}

1. Introduction. An amicable pair is a pair of positive integers $\left(m_{1}, m_{2}\right), m_{1} \neq m_{2}$, such that $\sigma\left(m_{1}\right)=\sigma\left(m_{2}\right)=m_{1}+m_{2}$, where $\sigma(\cdot)$ denotes the sum of divisors function. These number pairs have a long and interesting history (cf. [5]). Euler [4] was the first who systematically studied amicable pairs, and a great part of the known pairs were found with his methods and the use of electronic computers.

It is believed that there are infinitely many amicable pairs, although this has never been proved. In this note we present methods for finding new amicable pairs from given pairs. By applying these methods to a large list of known pairs, we have generated an average "offspring" of more than one "daughter" pair per given "mother" pair. We consider this result as strong numerical evidence for the existence of infinitely many amicable pairs.

In Section 2 we present one very simple method, and two methods derived from it, by which we have generated 1782 new amicable pairs from a list of 1575 known mother pairs. These mother pairs were collected from lists of Lee and Madachy ([5], 1108 pairs), Costello ([3], 7 pairs), Borho ([1], 3 pairs), Woods ([10], 456 pairs) and te Riele ([7], 1 pair). By a second (more restricted, cf. Section 3) application of the methods to the 400 smallest pairs in the list of 1782 daughter pairs mentioned above, still 88 more new "granddaughter" amicable pairs were generated. All these 1870 new pairs are explicitly given in [8], including their prime factorizations. It should be noted that other methods for finding new amicable pairs from known pairs are already known (so-called Thabit-rules, see [1]), but up till now relatively few new pairs have actually been found with these methods (cf. [1], [2], [6]).

In Section 3 we give details of our computations, including some information about the mother pairs from which the largest number of daughter pairs were generated.

This work was inspired by the following observations. By inspecting the Lee and Madachy-list we found that there are certain pairs $\left(m_{1}^{(i)}, m_{2}^{(i)}\right), i=1,2, \ldots, t, t \geqslant 2$,

Received October 18, 1982.

1980 Mathematics Subject Classification. Primary 10A40; Secondary 10A25, 10-04.

Ker words and phrases. Amicable pair. 
which share some common part, i.e., for which a pair of numbers $\left(a_{1}, a_{2}\right)$ exists with $a_{1}>1$ and $a_{2}>1$ such that $a_{1}$ is a divisor of $m_{1}^{(i)}$ with $\operatorname{gcd}\left(a_{1}, m_{1}^{(i)} / a_{1}\right)=1$ and $a_{2}$ is a divisor of $m_{2}^{(i)}$ with $\operatorname{gcd}\left(a_{2}, m_{2}^{(i)} / a_{2}\right)=1$, for $i=1,2, \ldots, t$. For example, the five pairs (which are numbered $1,3,4,9$ and 24, respectively, in [5]):

$$
\begin{aligned}
& \left\{\begin{array} { l } 
{ 2 2 0 = 2 ^ { 2 } 5 \cdot 1 1 } \\
{ 2 8 4 = 2 ^ { 2 } 7 1 }
\end{array} \left\{\begin{array} { l } 
{ 2 6 2 0 = 2 ^ { 2 } 5 \cdot 1 3 1 } \\
{ 2 9 2 4 = 2 ^ { 2 } 1 7 \cdot 4 3 }
\end{array} \quad \left\{\begin{array}{l}
5020=2^{2} 5 \cdot 251 \\
5564=2^{2} 13 \cdot 107
\end{array}\right.\right.\right. \\
& \left\{\begin{array} { l } 
{ 6 3 0 2 0 = 2 ^ { 2 } 5 \cdot 2 3 \cdot 1 3 7 } \\
{ 7 6 0 8 4 = 2 ^ { 2 } 2 3 \cdot 8 2 7 }
\end{array} \quad \left\{\begin{array}{l}
308620=2^{2} 5 \cdot 13 \cdot 1187 \\
389924=2^{2} 43 \cdot 2267
\end{array}\right.\right.
\end{aligned}
$$

share the common part $\left(a_{1}, a_{2}\right)=\left(2^{2} 5,2^{2}\right)$. Other examples are the five pairs numbered 6, 42, 90, 126 and 442 in [5] which share $\left(2^{3} 17,2^{3} 23\right)$ and the eight pairs numbered $8,19,20,29,121,213,260$ and 282 in [5] which share $\left(2^{4} 23,2^{4}\right)$.

Notation. We write $\bar{n}$ for $\sigma(n)$ and $\tilde{n}$ for $\sigma(n)-n$.

\section{The Methods.}

METHOD 1 (to find an amicable pair $\left.\left(a_{1} p_{1}, a_{2} q_{1} q_{2}\right)\right)$. Find a solution $\left(q_{1}, q_{2}\right)$ of the bilinear Diophantine equation

$$
D q_{1} q_{2}-\tilde{a}_{1} \bar{a}_{2}\left(q_{1}+q_{2}\right)=a_{1} \bar{a}_{1}+\tilde{a}_{1} \bar{a}_{2}, \quad D=a_{1} a_{2}-\tilde{a}_{1} \tilde{a}_{2},
$$

for which both $q_{1}$ and $q_{2}$ are primes, $q_{1} \neq q_{2}$, and $\operatorname{gcd}\left(a_{2}, q_{1} q_{2}\right)=1$. For such $a$ solution, compute $p_{1}$ from

$$
\tilde{a}_{1} p_{1}=a_{2} q_{1} q_{2}-\bar{a}_{1} .
$$

If $p_{1}$ is prime and $\operatorname{gcd}\left(a_{1}, p_{1}\right)=1$, then $\left(a_{1} p_{1}, a_{2} q_{1} q_{2}\right)$ is an amicable pair.

In fact, this method is well known (cf. [5, p. 81, formulas (12)-(15)]), but the difficulty is to find numbers $a_{1}$ and $a_{2}$ for which $q_{1}, q_{2}$ and $p_{1}$ are integral. Now we choose $a_{1}$ and $a_{2}$ as follows. Let $\left(m_{1}, m_{2}\right)$ be a known amicable pair and write it as $\left(a_{1} p, a_{2} q\right)$ where $p$ and $q$ are primes and $\operatorname{gcd}\left(a_{1}, p\right)=\operatorname{gcd}\left(a_{2}, q\right)=1$. By using the definition of an amicable pair, one easily verifies that $p$ and $q$ can be expressed in terms of $a_{1}$ and $a_{2}$ as follows:

$$
p=D^{-1}\left(\tilde{a}_{2} \bar{a}_{1}+a_{2} \bar{a}_{2}\right), \quad q=D^{-1}\left(a_{1} \bar{a}_{1}+\tilde{a}_{1} \bar{a}_{2}\right)
$$

where $D$ is defined in $(2.1)$.

So we have that $D$ is a divisor of $a_{1} \bar{a}_{1}+\tilde{a}_{1} \bar{a}_{2}$. Moreover, experiments have shown, that often $D$ is also a divisor of $\tilde{a}_{1} \bar{a}_{2}$, or at least that $D$ and $\tilde{a}_{1} \bar{a}_{2}$ have a large common divisor. This means that for this choice of $a_{1}$ and $a_{2}$ the coefficient of $q_{1} q_{2}$ in (2.1) can be made 1 (or at least a small integer $>1$ ) by dividing (2.1) by the greatest common divisor of $D, \tilde{a}_{1} \bar{a}_{2}$ and $a_{1} \bar{a}_{1}+\tilde{a}_{1} \bar{a}_{2}$. This is a favorable situation for the existence of integral solutions $q_{1}$ and $q_{2}$. Moreover, it turns out that often $p_{1}$ defined by (2.2) is integral when $q_{1}$ and $q_{2}$ are integral.

Example. If we choose $\left(a_{1}, a_{2}\right)=\left(2^{2} 5,2^{2}\right)$ from the smallest known amicable pair (see Section 1), then we have $D=14, \tilde{a}_{1} \bar{a}_{2}=154=11 \cdot 14$ and $a_{1} \bar{a}_{1}+\tilde{a}_{1} \bar{a}_{2}=994$ $=71 \cdot 14$. Equation $(2.1)$ then reads $q_{1} q_{2}-11\left(q_{1}+q_{2}\right)=71$, or, equivalently, $\left(q_{1}-11\right)\left(q_{2}-11\right)=192=2^{6} 3$. The solutions $\left(q_{1}, q_{2}\right)=(17,43)$ and $(13,107)$ give $p_{1}=131$ and $p_{1}=251$, respectively, so that with this method we have generated the (known) pairs numbered 3 and 4 in [5] (cf. Section 1). 
The following two methods are generalizations of Method 1, simply obtained by replacing $a_{1}$ by $a_{1} p_{2}$ (to give Method 2) and $a_{2}$ by $a_{2} q_{3}$ (to give Method 3). Here, $p_{2}$ resp. $q_{3}$ are "suitably" chosen primes (cf. (2.4) resp. (2.7) below).

METHOD 2 (to find an amicable pair $\left(a_{1} p_{1} p_{2}, a_{2} q_{1} q_{2}\right)$ ). Choose a prime $p_{2}$ such that $\operatorname{gcd}\left(a_{1}, p_{2}\right)=1$ and

$$
D p_{2}-\tilde{a}_{2} \bar{a}_{1}>0 \text {. }
$$

Find a solution $\left(q_{1}, q_{2}\right)$ of the bilinear Diophantine equation

$$
\begin{aligned}
\left(D p_{2}-\tilde{a}_{2} \bar{a}_{1}\right) q_{1} q_{2}-\left(\tilde{a}_{1} p_{2}+\bar{a}_{1}\right) \bar{a}_{2}\left(q_{1}+q_{2}\right) \\
=a_{1} \bar{a}_{1} p_{2}^{2}+\left(a_{1} \bar{a}_{1}+\tilde{a}_{1} \bar{a}_{2}\right) p_{2}+\bar{a}_{1} \bar{a}_{2}
\end{aligned}
$$

for which both $q_{1}$ and $q_{2}$ are primes, $q_{1} \neq q_{2}$, and $\operatorname{gcd}\left(a_{2}, q_{1} q_{2}\right)=1$. For such $a$ solution compute $p_{1}$ from

$$
\left(\tilde{a}_{1} p_{2}+\bar{a}_{1}\right) p_{1}=a_{2} q_{1} q_{2}-\bar{a}_{1}\left(p_{2}+1\right) .
$$

If $p_{1}$ is prime, $p_{1} \neq p_{2}$, and $\operatorname{gcd}\left(a_{1}, p_{1}\right)=1$, then $\left(a_{1} p_{1} p_{2}, a_{2} q_{1} q_{2}\right)$ is an amicable pair.

METHOD 3 (to find an amicable pair $\left(a_{1} p_{1}, a_{2} q_{1} q_{2} q_{3}\right)$ ). Choose a prime $q_{3}$ such that $\operatorname{gcd}\left(a_{2}, q_{3}\right)=1$ and

$$
D q_{3}-\tilde{a}_{1} \bar{a}_{2}>0 \text {. }
$$

Find a solution $\left(q_{1}, q_{2}\right)$ of the bilinear Diophantine equation

$$
\left(D q_{3}-\tilde{a}_{1} a_{2}\right) q_{1} q_{2}-\tilde{a}_{1} \bar{a}_{2}\left(q_{3}+1\right)\left(q_{1}+q_{2}\right)=a_{1} \bar{a}_{1}+\tilde{a}_{1} \bar{a}_{2}\left(q_{3}+1\right)
$$

for which both $q_{1}$ and $q_{2}$ are primes, $q_{1} \neq q_{2}, q_{1} \neq q_{3}, q_{2} \neq q_{3}$, and $\operatorname{gcd}\left(a_{2}, q_{1} q_{2}\right)=1$. For such a solution compute $p_{1}$ from

$$
\tilde{a}_{1} p_{1}=a_{2} q_{1} q_{2} q_{3}-\bar{a}_{1} .
$$

If $p_{1}$ is prime and $\operatorname{gcd}\left(a_{1}, p_{1}\right)=1$, then $\left(a_{1} p_{1}, a_{2} q_{1} q_{1} q_{3}\right)$ is an amicable pair.

Of crucial importance in Methods 2 and 3 is the choice of the primes $p_{2}$ resp. $q_{3}$. As with (2.1), $p_{2}$ and $q_{3}$ should be chosen such that the coefficient of $q_{1} q_{2}$ in (2.5) resp. (2.8) is as small as possible (preferably 1) after dividing by the greatest common divisor of the three coefficients.

3. Computational Details. We have applied Methods 1-3 to the 1575 mother amicable pairs mentioned in the introduction. For each of these pairs $\left(m_{1}, m_{2}\right)$ the following computations were carried out. We computed $g:=\operatorname{gcd}\left(m_{1}, m_{2}\right)$ and the quotients $m_{1} / g$ and $m_{2} / g$. From these quotients all possible pairs of primes $(p, q)$ were selected such that $\left(m_{1}, m_{2}\right)=\left(a_{1} p, a_{2} q\right)$ with $\operatorname{gcd}\left(a_{1}, p\right)=\operatorname{gcd}\left(a_{2}, q\right)=1$. To all the pairs $\left(a_{1}, a_{2}\right)$ (and $\left.\left(a_{2}, a_{1}\right)\right)$ obtained in this way, we applied Methods 1,2 and 3 with the following restrictions. Let the bilinear equations (2.1), (2.5) and (2.8) be written as

$$
c_{1} q_{1} q_{2}-c_{2}\left(q_{1}+q_{2}\right)=c_{3},
$$

with $\operatorname{gcd}\left(c_{1}, c_{2}, c_{3}\right)=1$. This is equivalent to

$$
\left(c_{1} q_{1}-c_{2}\right)\left(c_{1} q_{2}-c_{2}\right)=c_{1} c_{3}+c_{2}^{2}=: C \text {. }
$$


In those cases where $C$ was smaller than $10^{25}, C$ was completely factorized and for all possible products $C=B_{1} B_{2}$ the corresponding numbers $q_{i}=c_{1}^{-1}\left(c_{2}+B_{i}\right), i=$ 1,2, were computed, checked on being integral, checked on primality, etc. In Methods 2 and 3 we chose for $p_{2}$ resp. $q_{3}$ successively the smallest ten primes satisfying (2.4) resp. (2.7), in order to retain $c_{1}$ in (2.10) as small as possible.

In this way we have generated 1782 new amicable pairs from 1575 mother pairs, namely 170 with Method 1, 1523 with Method 2 and 89 with Method 3. Of course, also (relatively few) known pairs were generated. The smallest new pairs found with Methods 1,2 and 3 are, respectively:

$$
\begin{aligned}
& \left\{\begin{array} { l } 
{ 1 1 4 9 4 4 0 7 2 = 2 ^ { 3 } 1 7 \cdot 1 9 \cdot 4 4 4 8 3 } \\
{ 1 2 5 2 6 9 5 2 8 = 2 ^ { 3 } 5 3 \cdot 4 3 9 \cdot 6 7 3 }
\end{array} \text { with Method I from } \left\{\begin{array}{l}
726104=2^{3} 17 \cdot 19 \cdot 281 \\
796696=2^{3} 53 \cdot 1879
\end{array}([5, \# 37]) .\right.\right. \\
& \left\{\begin{array} { l } 
{ 3 2 3 4 0 1 7 1 2 = 2 ^ { 4 } 2 3 \cdot 4 3 1 \cdot 2 0 3 9 } \\
{ 3 3 2 2 7 0 6 0 8 = 2 ^ { 4 } 1 0 1 \cdot 1 2 7 \cdot 1 6 1 9 }
\end{array} \text { with Method } 2 \text { from } \left\{\begin{array}{l}
176272=2^{4} 23 \cdot 479 \\
180848=2^{4} 89 \cdot 127
\end{array}([5, \# 20]) .\right.\right. \\
& \left\{\begin{array} { l } 
{ 1 6 9 9 7 4 6 1 8 4 = 2 ^ { 3 } 2 3 \cdot 4 1 \cdot 2 3 3 \cdot 9 6 7 } \\
{ 1 7 2 5 1 1 5 2 5 6 = 2 ^ { 3 } 1 7 \cdot 1 2 6 8 4 6 7 1 }
\end{array} \text { with Method } 3 \text { from } \left\{\begin{array}{l}
10744=2^{3} 17 \cdot 79 \\
10856=2^{3} 23 \cdot 59
\end{array}([5, \# 6]) .\right.\right.
\end{aligned}
$$

The largest pair found is the pair of 38-digit numbers:

$$
\left\{\begin{array}{l}
84939420717490497547044056177577599145=E \cdot 359 \cdot 40939 \cdot 44296620189660299 \\
85084703583907612558249457600803200855=E \cdot 911 \cdot 119883499 \cdot 5971269576509 .
\end{array}\right.
$$

where $E=3^{6} 5 \cdot 19 \cdot 23 \cdot 137 \cdot 547 \cdot 1093$. generated with Method 2 from the pair $(E \cdot 359 \cdot 144779, E \cdot 911 \cdot 57149)$, which is pair \#1083 of [5].

From 533 of the 1575 mother pairs we actually generated one or more new daughter pairs. The three "champion" mother pairs are:

$$
\left\{\begin{array}{l}
77306245632044=2^{2} 11 \cdot 13 \cdot 47 \cdot 6829 \cdot 421079 \\
85036870195156=2^{2} 11 \cdot 1932656140799
\end{array}\right. \text { which is pair \#952 of [5], }
$$

from which 85 new pairs were generated with Method 2 .

$$
\left\{\begin{array}{l}
3693013664=2^{5} 41 \cdot 131 \cdot 21487 \\
3812143072=2^{5} 119129471
\end{array} \text { which is pair } \# 441\right. \text { of [5]. }
$$

from which 37 new pairs were generated with Method 2, and

$$
\left\{\begin{array}{l}
76809600128=2^{7} 149 \cdot 1151 \cdot 3499 \\
77414399872=2^{7} 604799999
\end{array}\right. \text { which is pair \#609 of [5], }
$$

from which 31 new pairs were generated with Method 2. We leave it to the reader to find out why in particular from these mother pairs so many daughter pairs could be generated. In [8] a frequency table is given consisting of all the mother pairs and the corresponding numbers of new daughter pairs generated from them with Methods 1,2 and 3.

In a second step, we applied our methods to the 400 smallest of the 1782 daughter pairs, with the restriction that per daughter pair $\left(m_{1}, m_{2}\right)$ we only considered one choice of $\left(a_{1}, a_{2}\right)$, viz., the pair obtained by dropping the largest prime divisor of $m_{1}$ from $m_{1}$ and the largest prime divisor of $m_{2}$ from $m_{2}$. As in our first step, we also considered only those cases for which $C<10^{25}$ in $\left(2.10^{\prime}\right)$. This reduced the number of cases drastically, also because the average daughter pair considered was much larger in size than the average mother pair considered, which led to much larger 
values of $C$ in $\left(2.10^{\prime}\right)$. Nevertheless, we found 88 new "granddaughter" pairs from the smallest 400 daughter pairs. These are also given in [8] together with a frequency table, similar to the one described above.

Finally, we have listed in [8] a compressed numbered list of all the 1575 mother pairs, for referencing and checking purposes.

Note added in proof. We have also applied our methods to 17 of 20 new amicable pairs given by Borho in [11], and we found 455 more new pairs in addition to the 1870 pairs mentioned above. The most "fertile" pair was the pair $\left(2 \cdot 5^{3} 19 \cdot 67\right.$. $\left.15959 \cdot 5346599,2 \cdot 5^{3} 19 \cdot 67 \cdot 85331735999\right)$, from which we found 147 new pairs. All 2325 new pairs are available from the author upon request.

Mathematical Centre

Kruislaan 413

1098 SJ Amsterdam. The Netherlands

I. W. BoRHo, "On Thabit ibn Kurrah's formula for amicable numbers," Math. Comp., v. 26, 1972, pp. $571-578$.

2. W. Borнo, "Some large primes and amicable numbers," Math. Comp., v. 36, 1981, pp. 303-304.

3. P. J. Costello, "Amicable pairs of Euler's first form," J. Recreational Math., v. 10, 1977-1978, pp. 183-189.

4. L. Euler, "De numeris amicabilibus," Leonhardi Euleri Opera Omnia, Teubner, Leipzig and Berlin, Ser. I, vol. 2, 1915, pp. 63-162.

5. E. J. LeE \& J. S. MADAChY, "The history and discovery of amicable numbers," J. Recreational Math., v. 5, 1972, part 1: pp. 77-93, part 2: pp. 153-173, part 3: pp. 231-249.

6. H. J. J. TE Riele, “Four large amicable pairs,” Math. Comp., v. 28, 1974, pp. 309-312.

7. H. J. J. TE RIELE, Further Results on Unitary Aliquot Sequences, Report NW 2/78, Mathematical Centre, Amsterdam, 2nd ed., Jan. 1978.

8. H. J. J. TE RIELE, Table of 1870 New Amicable Pairs Generated from 1575 Mother Pairs, Report NN 27/82, Mathematical Centre, Amsterdam, Oct. 1982.

9. D. Woods, "Construction of amicable pairs," Abstracts Amer. Math. Soc., v. 3, 1982, p. 223.

10. D. Woods, "List of 456 new amicable pairs," privately communicated to the author on June 29 , 1982.

11. W. Borho, "Grosse Primzahlen und befreundete Zahlen: über den Lucas-Test und Thabit-Regeln," Mitt. Math. Ges. Hamburg. (To appear.) 